\title{
Predictions of fracture toughness for weld material when adopting a novel particle failure criterion and a measured experimental particle distribution
} DOI:

10.1115/PVP2013-97509

Link to publication record in Manchester Research Explorer

Citation for published version (APA):

James, P., Ford, M., \& Jivkov, A. P. (2013). Predictions of fracture toughness for weld material when adopting a novel particle failure criterion and a measured experimental particle distribution. In American Society of Mechanical Engineers, Pressure Vessels and Piping Division (Publication) PVP/ASME Pressure Vessels Piping Div. Publ. PVP (Vol. 6). American Society of Mechanical Engineers. https://doi.org/10.1115/PVP2013-97509

\section{Published in:}

American Society of Mechanical Engineers, Pressure Vessels and Piping Division (Publication) PVP|ASME Pressure Vessels Piping Div. Publ. PVP

\section{Citing this paper}

Please note that where the full-text provided on Manchester Research Explorer is the Author Accepted Manuscript or Proof version this may differ from the final Published version. If citing, it is advised that you check and use the publisher's definitive version.

\section{General rights}

Copyright and moral rights for the publications made accessible in the Research Explorer are retained by the authors and/or other copyright owners and it is a condition of accessing publications that users recognise and abide by the legal requirements associated with these rights.

\section{Takedown policy}

If you believe that this document breaches copyright please refer to the University of Manchester's Takedown Procedures [http://man.ac.uk/04Y6Bo] or contact uml.scholarlycommunications@manchester.ac.uk providing relevant details, so we can investigate your claim.

\section{OPEN ACCESS}




\section{PREDICTIONS OF FRACTURE TOUGHNESS FOR WELD MATERIAL WHEN ADOPTING A NOVEL PARTICLE FAILURE CRITERION AND A MEASURED EXPERIMENTAL PARTICLE DISTRIBUTION}

\author{
Peter James \\ AMEC, Clean Energy Europe \\ Risley, Warrington, UK
}

\author{
Mike Ford \\ AMEC, Clean Energy Europe \\ Risley, Warrington, UK
}

\author{
Andrey P Jivkov \\ The University of Manchester \\ Manchester, UK
}

\begin{abstract}
It is important for safety case justification of the continued use of nuclear power plant that any changes in the ferritic RPV steels' fracture toughness with temperature, irradiation and geometry can be accounted for, particularly with regards to plant life extension. It has been demonstrated that local approach methods have the potential to provide such estimates by assessing the likelihood of cleavage rupture.

Here a micro-structurally informed, post-processing, local approach methodology with a new rupture criterion is presented. This has been applied to a base and weld material pair, made available under the EU FP7 PERFORM 60 Programme. This material has been selected as tensile and fracture data are available along with results of recently performed analyses to characterise and quantify the size distribution of fracture initiators for the weld material (predominantly found to be alumino-silicates) which is used as an input to the model.

A series of finite element analyses have been performed for two-dimensional three-point bend specimens over a range of temperatures, constraint and irradiation states. Application of the local approach model to these results has then been favourably compared to the experimentally determined toughness. This has been achieved for a range of conditions when using the experimentally determined initiator particle distribution and maintaining the same calibration terms throughout for each material.
\end{abstract}

\section{INTRODUCTION}

Safety assessment and life extension decisions in nuclear plant require reliable methodologies for predicting changes in cleavage fracture toughness behaviour of ferritic reactor pressure vessel (RPV) steels due to irradiation and defect geometry effects. Local approaches (LA) to cleavage fracture are promising as these could account for the micro-mechanisms involved in the cleavage failure phenomenon, such as the nucleation of micro-cracks at second-phase brittle particles, the propagation of such micro-cracks within grains and the propagation of a critical micro-crack leading to component failure [1]. The pioneering LA to cleavage, proposed by the Beremin group [2], is based on two main assumptions: that the global failure probability is a weakest-link event and that the individual failure probabilities are dictated by local mechanical fields and specific microstructure data such as the size distribution and number density of cleavage-initiating particles. Assuming that the tail of the size distribution can be approximated by a power-law, the weakest-link statistics leads to a global failure probability expressed as a Weibull-type function of a generalised stress, the Weibull stress $\sigma_{w}[2,3]$ :

$P_{f}(V)=1-\exp \left[-\left(\frac{\sigma_{w}}{\sigma_{u}}\right)^{m}\right]$

The shape, $m$, and scale, $\sigma_{u}$, of the Weibull function can be calibrated using fracture toughness data obtained with particular crack geometry at a given temperature and corresponding FE analysis [4]. For calibrations performed with high-constraint crack geometries at or below the ductile-tobrittle transition temperature, $T_{0}$, the shape and scale obtained can be used with sufficiently good accuracy to predict cleavage fracture toughness of other high-constraint geometries at temperatures below $T_{0}$ [2-4]. However, an attempt to use the same parameters to calculate cleavage toughness under lower constraint conditions or higher temperatures leads to predictions that do not match experimental values. For cleavage fracture toughness data obtained at a given temperature with low- and high-constraint geometries, independent calibrations 
would typically show that the low-constraint $m$ is smaller than the high-constraint $m$ (assuming that $\sigma_{u}$ is not dependent on constraint), see e.g. [5]. This suggests that the excess of plastic deformations introduced by the lower constraint require reduction of $m$ to make reliable predictions. The methodology proposed in [5] for cross-calibration of $m$ between high and low constraint geometries has shown promise [5, 6], but remains limited to temperatures well below $T_{0}$, where the lower constraint conditions do not introduce substantial increase in plastic deformations relative to the high constraint case. This may prove problematic when used to predict cleavage fracture toughness for short cracks in irradiated material, where the reference temperature is shifted to higher values. To predict the irradiation effects on cleavage one needs a reliable model for the ductile-to-brittle transition (DBT) regime. Here, however, the Weibull parameters need to be varied to match experimental data. One possibility is to keep the shape parameter constant, which leads to temperature dependence of the scale, $\sigma_{u}[7,8]$. Another possibility is to keep the scale parameter constant, which leads to temperature dependence of the shape, $m[9,10]$. As for the low-constraint situation, $m$ needs to be reduced with increasing temperature, i.e. with enhanced plasticity.

Considering the physical basis for the Beremin LA [2, 3], the current state of affairs is not satisfactory, because the Weibull parameters must depend exclusively on the material microstructure. If the model for individual failure probability accounted adequately for the local fields and microstructure effects on particle failure, and the global failure were a weakest-link event, then the changes in plasticity due to constraint or temperature increase should be already accounted for, leaving the Weibull parameters constants. In particular, $m$ should be linked to the shape of size distribution of cleavage initiation particles, while $\sigma_{u}$ should depend on the elastic properties and surface energy of the material as well as on the scale of the particle size distribution. Since these parameters do not change noticeably with constraint or temperature, the need to vary $m$ and $\sigma_{u}$ at increasing plastic deformations suggests that the link between physics and mechanics breaks. One possibility is that the individual failure probability model does not account adequately for the mechanical and particle size effects. A second possibility is that with the increase of plastic deformations the population of micro-cracks that needs to be accounted for in the weakest-link statistics becomes larger than the tail of the distribution, approximated by power-law in the Weibull-stress models. A third possibility is that the weakestlink assumption becomes increasingly invalid with increasing plasticity and micro-crack interaction effects need to be accounted for.

To improve the individual failure probability, the effect of the plastic strain has been introduced in modifications of the Beremin model $[11,12]$ as well as in incremental formulations [13]. Recently, in addition to plastic strains the effect of stress triaxiality has also been introduced [14]. In a previous work [15], we have compared these models with the original Beremin and demonstrated that they provide improvements in the predicted failure probability profiles ahead of cracks with different constraints. This comparison has been done against a large set of experimental data for the locations of cleavage initiation sites reported in [16]. However, the prediction of the cleavage fracture toughness temperature dependence with these models remained unsatisfactory when performed with single set of Weibull parameters calibrated at $T_{0}$. The effect of particle size on local failure probability was first proposed in [17]. The model now known as WST, however, remained as a microstructure-informed local model and to our knowledge has not been applied for global failure predictions. A different direction of work considers ductile damage prior to cleavage in the DBT regime, employing damage models for the material behaviour coupled with subsequent calculation of failure probability following Beremin $[18,19]$. This approach has the potential to capture better the fracture behaviour in the DBT, but the models are presently too simplified with no interaction of void growth and micro-cracking at microstructure level [20]. Until advanced coupled models are developed and tested, the wider engineering community would benefit from a simpler uncoupled cleavage fracture model for the DBT region.

In [15] we have proposed a model incorporating mechanical and particle size effects and demonstrated that it provides excellent predictions for failure probability profiles. In this work we report on application of the model to predict cleavage fracture toughness in DBT regime. We focus on the first two possibilities mentioned above: to improve individual probability of failure and to account directly for the real particle size distribution rather than approximating the tail. The validity of the weakest-link assumption for the calculation of global probability is maintained.

\section{THEORY AND MODEL Statistical Basis}

LA methods share a common philosophy based on two distinct components. Firstly, the local mechanical fields provide a local or 'individual' probability of failure when linked to the size distribution of the micro-crack initiators. The individual probability of failure at location $i$ is

$$
p_{f, i}=\int_{r_{c, i}}^{\infty} p_{c, i} f(r) d r
$$

where $f(r)$ is the probability density of initiators' sizes, $p_{c, i}$ is the probability of micro-crack nucleation, and $r_{c, i}$ is a critical micro-crack size at location $i$. Note, that $p_{c} f(r)$ is the probability density of nucleated micro-crack sizes. Existing LAs can be recast into Eq. (2) albeit with different definitions of $p_{c}$ and $r_{c}$. For example in [2-4] $p_{c}=0$ or 1 for zero and 
non-zero plastic strains. In $[11,12] p_{c}$ scales with the equivalent plastic strain, while in [14] $p_{c}$ scales with the equivalent plastic strain and exponent of the stress triaxiality. In [13] $p_{c}$ is a more complex function of stress and plastic strain increments. In all cases $r_{c}$ is defined via Griffith or plasticitymodified Griffith criterion. Common feature of [2-4, 11-14] is that $p_{c}$ is independent of particle size.

Secondly, it is assumed that the individual failure events are independent. This allows the weakest-link argument to be invoked for calculating the global failure probability, so that

$$
P_{f}(V)=1-\prod_{i=1}^{N}\left(1-p_{f, i}\right)
$$

where $N$ is the number of possible weakest-links, i.e. active micro-cracks, in volume $V$. In practice, LAs are applied to FE solutions of cracked components, where the mechanical fields are constant within an integration point volume, $V_{i}$. The failure probability of such a volume is thus

$$
P_{f}\left(V_{i}\right)=1-\left(1-p_{f, i}\right)^{N_{i}}=1-\left(1-p_{f, i}\right)^{\rho_{i} V_{i}}
$$

where $N_{i}=\rho_{i} V_{i}$ is the number of micro-cracks in $V_{i}$, and $\rho_{i}$ is the density of the micro-cracks. Strictly speaking $\rho_{i}=\rho p_{c, i}$, where $\rho$ is the density of initiating particles in the material. The component probability of failure is then calculated by repeated application of (3) to get

$P_{f}(V)=1-\prod_{i=1}^{I P}\left(1-p_{f, i}\right)^{\rho_{i} V_{i}}$

where the product is taken over all integration points. When $p_{c}$ is independent of particle size, Eq. (2) can have a closedform solution in terms of critical micro-crack sizes, $r_{c}$. With a power law approximation for particle sizes and assuming small individual failure probabilities, Eq. (5) yields the Weibull-stress function in Eq. (1). We use Eq. (5) directly to calculate the global failure probability, because the experimental particle size distribution, given in Section 2.2, and our expression for individual failure probability, derived in Section 2.3, do not allow for closed form solution of Eq. (2) and hence a corresponding definition of a generalised stress.

\section{Experimental Data}

The material analysed here is a ferritic base and weld pairing for a German pressure vessel steel, as has been used in their Konvoi pressurized water reactor. This data has been made available for use in the European FP7 project PERFORM 60 . The base metal is a $20 \mathrm{MnMoNi} 5$ ferritic steel, similar to an A533B class 1 steel. The weld material is for a
S3NiMo1/OP41 TT submerged-arc weld. The data available from the PERFORM 60 database [21] for these two materials include the mechanical and fracture toughness properties over a range of temperatures and irradiation states within the lower shelf and in the DBT. The fracture toughness data is only available under high constraint conditions in the unirradiated state, but is available in both high and low constraint conditions for the irradiated state. Details of the materials Young's Modulus were not available, so typical temperature dependent properties for ferritic steels were used as in Eq. (6) below.

$$
E=-90 T+206000
$$

The temperature dependence of the proportionality stress $\sigma_{0}$ and ultimate tensile strength $\sigma_{u}$ for the base metal under unirradiated conditions are:

$$
\begin{aligned}
& \sigma_{0}=347.4+58.5 \exp (-T / 88.2) \\
& \sigma_{u}=511.3+98.1 \exp (-T / 126.2)
\end{aligned}
$$

where $T$ is in ${ }^{\circ} \mathrm{C}$ and $E, \sigma_{0}$, and $\sigma_{u}$ are in MPa. Poisson's ratio is $v=0.3$ independent of temperature. Likewise, the temperature dependence of $\sigma_{0}$ and $\sigma_{u}$ for the unirradiated weld metal:

$$
\begin{aligned}
& \sigma_{0}=447.4+47.7 \exp (-T / 81) \\
& \sigma_{u}=555.8+49.6 \exp (-T / 89.4)
\end{aligned}
$$

Under the irradiated state considered here (an average fluence of $\sim 8 \times 10^{18} \mathrm{n} \mathrm{cm}^{-2}$, En $\left.>1 \mathrm{MeV}\right) E$ is assumed to remain unchanged and $\sigma_{0}$ and $\sigma_{u}$ are redefined as in Eq. (9) for the base metal and Eq. (10) for the weld material.

$$
\begin{aligned}
& \sigma_{0}=363.4+96.1 \exp (-T / 116.6) \\
& \sigma_{u}=524.7+94.0 \exp (-T / 127.3) \\
& \sigma_{0}=470.9+105.0 \exp (-T / 126.1) \\
& \sigma_{u}=567.5+92.4 \exp (-T / 130.4)
\end{aligned}
$$

The fracture toughness properties of the base metal and weld pairing given in [21] were determined according to the ASTM standard [22] using SEN(B) specimens in three-point bending. This standard is based on the Master Curve formalism, which defines the temperature dependence of a reference toughness, $K_{0}$, relative to a reference temperature, $T_{O}$, at which $K_{O}=108 \mathrm{MPa} \sqrt{\mathrm{m}}$, for high-constraint cracked geometries with reference crack front length $B_{0}=25.4 \mathrm{~mm}$

$K_{O}=31+77 \exp \left[0.019\left(T-T_{0}\right)\right]$ 
The scatter in measured cleavage fracture toughness values is described as a function of the probability of failure, $p$, and the actual crack front length, $B$, with

$$
K_{p}=K_{\text {min }}+\left(K_{0}-K_{\min }\right)\left(\frac{B_{0}}{B}\right)^{1 / 4}\left(\ln \frac{1}{1-p}\right)^{1 / 4}
$$

where $K_{\min }$ is a temperature independent threshold toughness, $K_{\text {min }}=20 \mathrm{MPa} \sqrt{\mathrm{m}}$ in [22]. Eqs. (11) and (12) define the cleavage fracture toughness behaviour of a material with known $T_{0}$. The reference temperature for deep-notch $\left(a_{0} / W\right.$ $=0.5)$ specimens has been calculated at $T_{0}=-75.6^{\circ} \mathrm{C}$ for the base material and $-62.7^{\circ} \mathrm{C}$ for the weld material. In the present work, we assume that Eqs. (11) and (12) provide a relevant representation of experimental data and assess our model against it. Nevertheless, experimental data points are given in the results together with Master Curve and model predictions.

The density and size distribution of cleavage initiating particles in a similar ferritic pressure vessel base metal (Material A) have been reported in [23]. For Material A the nature of the initiators has been determined by fractography revealing that cleavage in this material initiated predominantly at metal carbides, specifically M3C and M23C6, and occasionally at carbide-sulphate composites. Comprehensive metallographic examination of carbides provided number density $\rho=7.6 \times 10^{17} \mathrm{~m}^{-3}$ and a probability density of particle sizes which was fit by

$$
f(r)=\frac{\beta}{r_{0}}\left(\frac{r}{r_{0}}\right)^{-\beta-1} \exp \left[-\left(\frac{r}{r_{0}}\right)^{-\beta}\right]
$$

with $\beta=2.7$ being a shape parameter, and $r_{0}=0.036 \mu \mathrm{m}$ being a scale parameter representing the mean particle size. In this work, we use this probability density distribution directly for calculating the local probability of failure via Eq. (2) for the base material. It is accepted that there is a likely difference in the mean particle size and shape parameter between Material A and the base material used here, but without further information this data has been used as a first approximation. For future work it would be beneficial to consider actual measured data (if this becomes available), or alternatively to consider a sensitivity analyses on the mean particle size and shape parameter.

More detail is, however, available for the weld material. Fractography of the weld material [24] has revealed that cleavage in this material initiated predominantly at manganesealumino-silicates (with traces of $\mathrm{Mg}, \mathrm{S}$, and $\mathrm{Ti}$ ) and at complex mixed inclusions. Clearly this is different to what might nominally be expected for the base material and other ferritic steels (such as Material A) where the initiating particle would be expected to be carbides predominantly. Comprehensive metallographic examination of initiating particles has also been performed for the weld material [25] that showed the planar unit area number density to be $8.3 \times 10^{9} \mathrm{~m}^{-2}$ which can be correlated to a number density of $\rho=7.6 \times 10^{14} \mathrm{~m}^{-3}$. The size distribution was found to be best fit with a logistic function (when considering the cumulative probability) as illustrated in Figure 1 below.
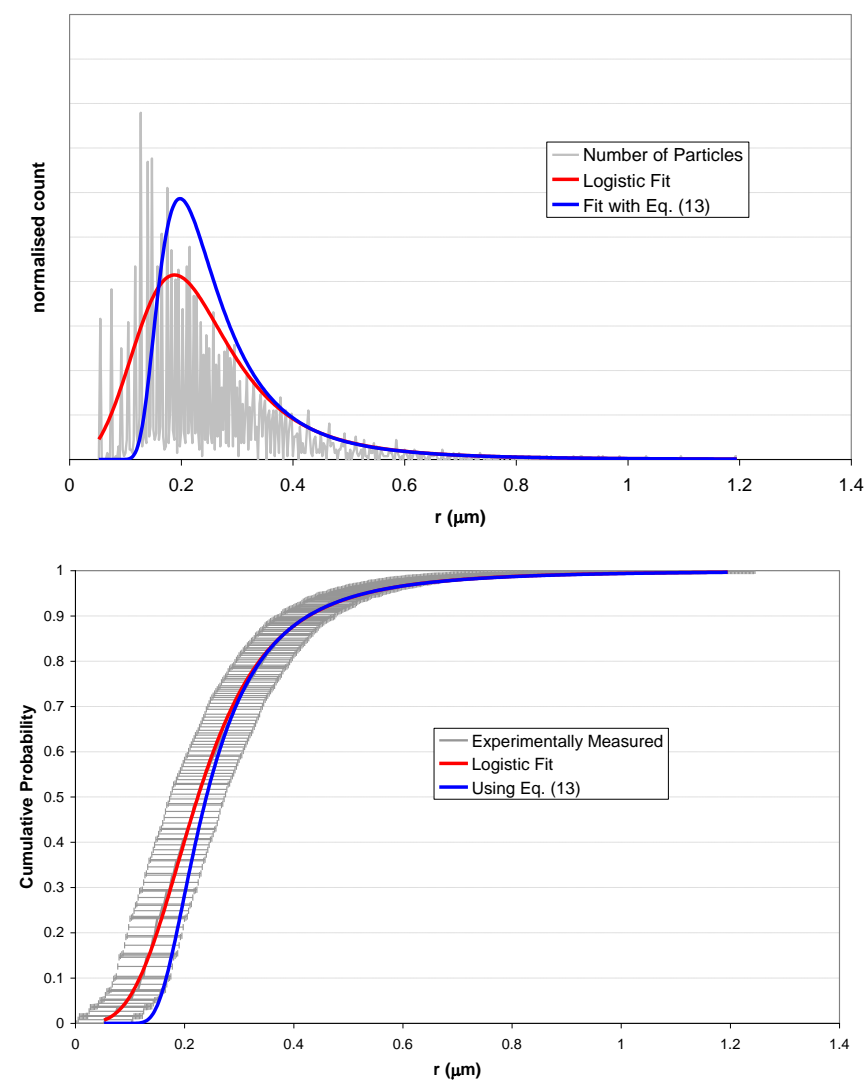

FIGURE 1 - COMPARISON OF MEASURED PARTICLE SIZE DISTRIBUTION FOR PROBABILITY DISTRIBUTION (TOP) AND CUMULATIVE PROBABILITY (BOTTOM) COMPARED TO LOGISTIC FIT AND EQUATION (13)

However, the tail of logistic distribution was well characterised by Eq. (13) as shown in Figure 1. The fit uses $\beta=3.3$ and $r_{0}=0.21 \mu \mathrm{m}$, and allowed for the continued use of Eq. (13) whilst still providing a good description of the tail of the size distribution. This will mean that, as long as only large particles are required to rupture, the results should be suitable.

\section{Micro-Mechanically Informed Model}

The expression for the probability of particle failure is based on experimental observations that this probability depends not only on the mechanical fields but also on the particle size, similarly to the WST model [17]. The rationale is that larger particles have higher probability of failure than 
smaller particles under identical mechanical conditions. The particles are assumed to be elastic-brittle with elastic constants equal to those of the matrix as a first approximation. The principle stresses, $\Sigma_{\alpha}$, in a particle can be given in terms of the principal stresses, $\sigma_{\alpha}$, and plastic strains, $\varepsilon_{\alpha}^{p}$, in the matrix with

$\Sigma_{\alpha}=\sigma_{\alpha}+\frac{E}{1+v} \varepsilon_{\alpha}^{p}, \quad \alpha=1,2,3$

The criterion of particle failure is based on a critical value of the strain energy density in a particle associated with failure normal to the maximum principal stress. This is given by

$\psi_{c}=\frac{1+v}{E} \Sigma_{I}^{2}-\frac{v}{E} \Sigma_{I} \Sigma_{h}$

where $\Sigma_{I}$ and $\Sigma_{h}\left(=\sigma_{h}\right)$ are the maximum principal and the hydrostatic stress in the particle, respectively. If a particle of size $r$ ruptures normally to the maximum principal stress upon achieving some critical condition, the energy lost (or the work of rupture) will be proportional to $\Psi_{c}=r^{3} \psi_{c}$. The rupture of a particle is a stochastic event governed by the work of rupture and depending on the unknown particle constitution. The survival probability, $p_{s}$, of the particle must decrease with increasing work of rupture, which can be written in the form

$\frac{d p_{s}}{p_{s}}=-\frac{d \Psi_{c}}{\Psi_{0}}$

where $\Psi_{0}$ is a scaling energy. The survival probability at a given $\Psi_{c}$ can be determined by integrating Eq. (16) from the initial value of the work of rupture, which is zero, to the current value. The probability of particle rupture, $p_{c}$, is one minus the survival probability

$p_{c}=1-\exp \left(-\frac{\Psi_{c}}{\Psi_{0}}\right)=1-\exp \left[-\left(\frac{r}{r_{0}}\right)^{3} \frac{\psi_{c}}{\psi_{0}}\right]$

where the rupture energy density scale $\psi_{0}=\Psi_{0} / r_{0}^{3}$ is introduced for convenience. Note, that Eq. (17) incorporates the effects of plastic strain and stress triaxiality. Increased plastic strains in the matrix will result in increased $\psi_{c}$ and hence probability of micro-crack formation, with all other conditions equal. Inversely, increased hydrostatic stress, which could lead to micro-crack blunting, will result in reduced $\psi_{c}$ and hence probability of micro-crack formation under equal other conditions, see Eqns. (14) and (15).
We use Eq. (17) and Eq. (13) into Eq. (2) to calculate individual probabilities of failure at the integration points by numerical integration. The lower limit of the integral, i.e. the critical micro-crack size at given point, is defined with a heuristic argument leading to a new modification of the Griffith criterion to account for plasticity. We assume that the behaviour of a micro-crack of radius $r$ formed in the plastic matrix corresponds to a fictitious micro-crack of radius $r_{f}>r$ in an elastic matrix. The crack opening displacement of a pennyshaped crack of radius $r_{f}$ in an elastic material subject to normal stress $\sigma_{I}$ is given by

$u(x)=\frac{4\left(1-v^{2}\right) \sigma_{I}}{\pi E} \sqrt{r_{f}^{2}-x^{2}}, \quad 0<x<r_{f}$

The blunting of the physical micro-crack formed after particle rupture can be approximated by $r \varepsilon_{I}^{p}$. The fictitious micro-crack size is then defined in such a way that the opening at $x=r$ equals the blunting of the physical micro-crack, i.e.

$u(r)=r \varepsilon_{I}^{p}$. Solving Eq. (18) with this constraint leads to the following expression for the fictitious micro-crack size

$r_{f}=r \sqrt{1+\left[\frac{\pi E \varepsilon_{I}^{p}}{4\left(1-v^{2}\right) \sigma_{I}}\right]^{2}}$

Used in conjunction with the Griffith criterion an effective critical micro-crack size can be defined that accounts for the crack tip blunting as

$r_{c}=\frac{\pi E \gamma_{s}}{2\left(1-v^{2}\right) \sigma_{I}^{2}} \sqrt{1+\left[\frac{\pi E \varepsilon_{I}^{p}}{4\left(1-v^{2}\right) \sigma_{I}}\right]^{2}}$

The square-root factor in Eq. (20) replaces the exponent of plastic strain in the modified Griffith criterion used previously [2-14]. As before, it is intended to account for the reduction in crack driving force due to plastic dissipation in the matrix (i.e. crack tip blunting). The difference is that in the case of Eq. (20) the effect is not only dependent on the plastic strain but also on stress triaxiality via the ratio between the maximum plastic strain and the maximum stress.

\section{FE Model}

A three-point bend specimen was used in the finite element analyses to which the developed LA model has here been applied as this most closely resembles the Pre-Cracked Charpy$\mathrm{V}$ (PCCV) specimens used in the tests. Two crack depths were considered to represent both high and low constraint 
conditions; with crack depth of $a / t=0.5$ and 0.2 respectively. The three point bend specimens used were $30 \mathrm{~mm}$ through thickness by $200 \mathrm{~mm}$ long and nominally $25.4 \mathrm{~mm}$ deep to match the validity of the fracture tests (although they are modelled as a unit thickness under 2-dimensional plane strain conditions). Non-deforming, rigid, loading rollers were positioned with a total span of $180 \mathrm{~mm}$ (i.e. $10 \mathrm{~mm}$ from the edges of the specimen) and at the centre crack back-face. The finite element model to describe this geometry was created and run within ABAQUS 6.11 [26] and was composed of 34,000 CPE8R 2-dimensinal elements. The crack tip region was modelled as a fine blunt notch with a crack-tip radius of $25 \mu \mathrm{m}$ for use in large strain analyses. The crack tip elements were modelled as $5 \mu \mathrm{m}$ long and $0.6 \mu \mathrm{m}$ wide. An illustration of the finite element model can be seen in Fig. 1 as the global model which also incorporates the crack tip region.

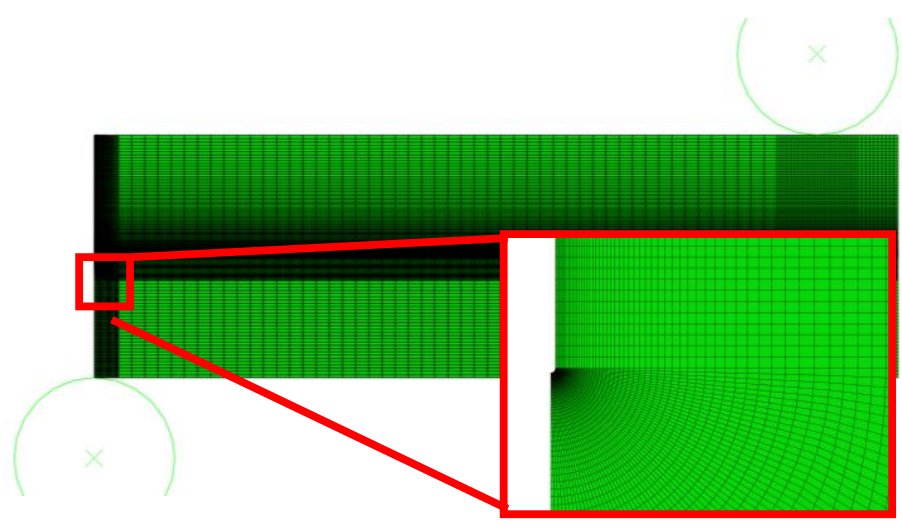

\section{FIGURE 2 - FE MODEL OF 3PB SPECIMEN}

The primary loading was applied under displacement control conditions by displacing the bottom loading pin into the specimen whilst preventing the top pin from moving. The symmetry of the geometry and loading allows the un-cracked ligament to act as the symmetry plane. It was also necessary to apply boundary conditions to the loading pins to prevent free rotation and unwanted displacement. Standard contact was used with a small adjustment of $0.015 \mathrm{~mm}$ to the mesh around the rigid surface of the loading pins to ensure correct transference of the load. The contact was also modelled as having no separation, ensuring contact throughout the analyses.

In each analysis the estimate of the $J$-contour integral was used to provide the value of crack tip stress intensity factor (which was extracted as part of the post-processing routine used in the local approach prediction of failure). It is noted that the validity of $J$ for these cases can be questioned as the crack tip was a blunt notch and the applied load caused the plastic zone to reach the back face and interact with the loading pin, preventing the contour zone from fully encompassing the fracture process zone (plastic zone). However, it is noted that the blunt notch was used to accommodate large strain analyses with large deformations at the crack tip and that the plastic zone can become very large. To try and encompass as much of the plastic zone as possible up to 80 contours were taken away from the crack tip node set (i.e. those on the blunt tip). By taking 80 contours the estimate of $J$ covered as much of the plastic zone as possible whilst remaining contained in the model. Validation of the model was performed that provided confidence in the model, loading and extracted values of $J$. Values of the $J$-contour integral were taken at each loading increment in the analyses and converted to a value of $K$. All estimates of $J$ were taken at the deepest point of the crack.

The materials used in the finite element analyses were based upon the material cases presented in Section 2.2 over the range of temperatures in the irradiated and unirradiated states. To interpret the yield stress and ultimate tensile strength within the analyses at a given temperature a power-law relation was used for the uniaxial stress, $\sigma$, and strain, $\varepsilon$, which remained elastic below yield and was dependent on $\sigma^{n}$ after yielding. The determination of the power law exponent, $n$, has been made using the Considère's rule describing the stress-strain condition at the point of loss of stability, represented by $\sigma_{U T S}$ in engineering stress terms. For both irradiation conditions a range of temperatures were assessed to cover the transition curve. These were generally spaced between approximately $\pm 60{ }^{\circ} \mathrm{C}$ of the high constraint un-irradiated $T_{0}$ temperature in a maximum of $10{ }^{\circ} \mathrm{C}$ increments.

\section{RESULTS AND DISCUSSION}

The model allows a number of parameters to be calibrated depending on knowledge of the material being assessed. Here information on the carbide distribution for the base material was inferred from similar material (i.e. $\beta=2.7$ and $r_{0}=0.036$ $\mu \mathrm{m})$ for the parent material. For the weld material a measured distribution is available as detailed above for the aluminosilicate initiating particles. Having an estimate of these distributions for these materials leaves the rupture energy density scale, $\psi_{0}$, and the materials surface energy, $\gamma_{s}$, to be calibrated. It is noted that typical values of $\gamma_{s}$ for ferritic steels can be inferred from the theoretical values for $\mathrm{Fe}$ [27] and are in the order of approximately 2.0 to $2.4 \times 10^{-3} \mathrm{KJm}^{-2}$.

For the calibration of the base metal it was found that a value of $\gamma_{s}$ greater than $1.5 \times 10^{-3} \mathrm{KJm}^{-2}$ was not able to provide accurate predictions irrelevant of the value of $\psi_{0}$ used. Therefore a value of $10^{5}$ was adopted for $\psi_{0}$. As demonstrated in [28] this value provided good comparisons to Material A toughness and sites of cleavage initiation. Therefore the only parameter to calibrate was $\gamma_{s}$. This was determined so that the individual probability of failure profile ahead of the crack corresponded to a global $50 \%$ probability of cleavage at $T_{O}$ with an applied $K$ of $100.3 \mathrm{MPa} \sqrt{\mathrm{m}}$ (i.e. defined from the experimentally determined Master Curve). On calibration by varying $\gamma_{s}$ to match the $T_{0}$ toughness on the unirradiated high 
constraint case, $\gamma_{s}$ was found to be $1.1 \times 10^{-3} \mathrm{KJm}^{-2}$. This value is somewhat lower than what might be expected for this material but is still believed to be close enough considering some of the assumptions adopted here for material properties and carbide distribution.

When calibrating the weld material case, neither the value of $\gamma_{s}$ nor $\psi_{0}$ were known. Therefore two calibrations were made when making educated guessed for $\gamma_{s}$ and $\psi_{0}$ respectively. When calibrating against $\gamma_{s}, \psi_{0}$ was held to be $10^{5}$, as used above, and $\gamma_{s}$ was found to be $2.9 \times 10^{-3} \mathrm{KJm}^{-2}$. Conversely when calibrating against $\psi_{0}$, the value of $\gamma_{s}$ was approximated to be $2.2 \times 10^{-3} \mathrm{KJm}^{-2}$ and $\psi_{0}$ was found to be $1.9 \times 10^{6}$. It is noted here that the results were found to be relatively insensitive to $\psi_{0}$, hence the larger respective change. It is considered that the calibrated values of $\gamma_{s}$ show reasonably good agreement with the values expected on theoretical grounds.

The predictive bounds from the model compared to both experimental data and the Master Curve comparison is provided in Fig. 3 for the unirradiated base material. In the figure (as in all figures below) the red curves represent the model predictions at 5\%,50\% and $95 \%$ probability bounds, the black curves are the Master Curve predictions at 5\%, 50\% and 95\% probability bounds and experimental data are the green diamonds. The plots are provided as estimates of $K$ at failure over a range of temperatures, shown as $\Delta T\left(\Delta T=T-T_{0}\right)$, where $T_{0}$ is taken for the unirradiated material in all cases so that any shifts in fracture toughness are apparent.

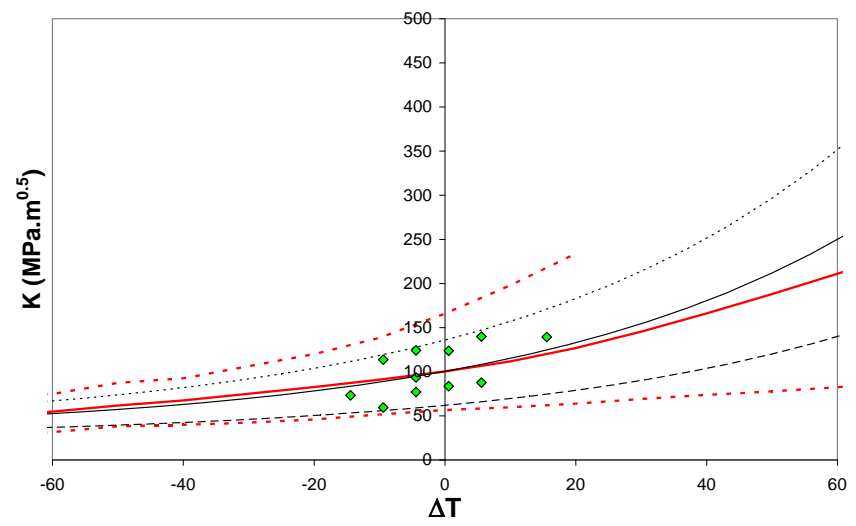

FIGURE 3 - TEMPERATURE DEPENDENCE PREDICTED BY PROPOSED MODEL FOR THE UNIRRADIATED HIGH CONSTRAINT BASE MATERIAL COMPARED TO EXPERIMENTAL DATA AND THE MASTER CURVE.

It can clearly be seen that the model presented here provides a good fit of the experimental data with all experimental points within the probability bounds, which was also observed for the Master Curve. The comparison to the Master Curve is slightly tighter to the experimental data but in general the results are very encouraging. It is also noted, as the carbide distribution is only inferred here, that if the value of $\beta$ is increased to 4 the results essentially follow the Master Curve. The accuracy of the model here also helps provide confidence in the finite element analyses and implementation of the developed local approach model.

When progressing to the irradiated high constraint material, the corresponding results can be seen in Fig. 4 below.

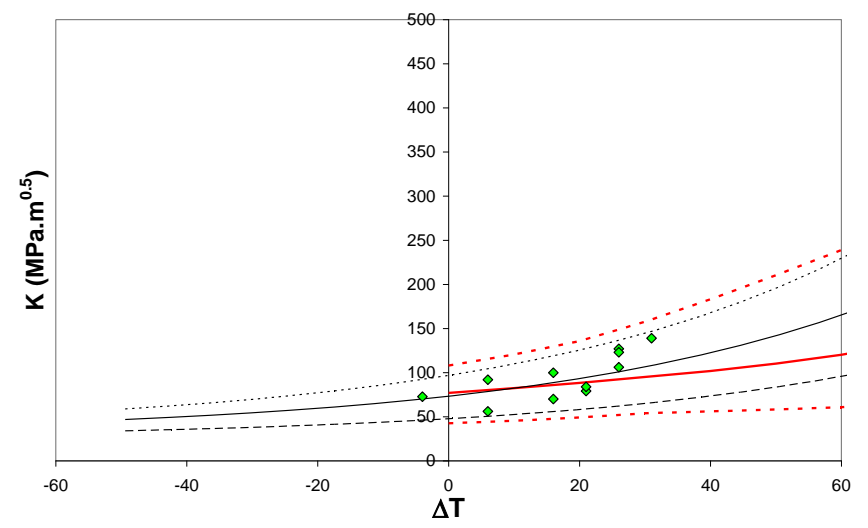

FIGURE 4 - TEMPERATURE DEPENDENCE PREDICTED BY PROPOSED MODEL FOR THE IRRADIATED HIGH CONSTRAINT BASE MATERIAL COMPARED TO EXPERIMENTAL DATA AND THE MASTER CURVE.

Again, it can clearly be seen that the model presented here provides a good fit of the experimental data with all experimental points within the probability bounds, which, again, was also observed for the Master Curve. Here an inferred shift in toughness at an applied $\mathrm{K}$ of $100.3 \mathrm{MPa} \sqrt{\mathrm{m}}$ (i.e. as measured in Master Curve shifts) would be approximately $+40 \mathrm{oC}$, which is greater than that from the Master Curve of just over +20 oC. This difference may be linked to a flattening of the $5 \%$ and $50 \%$ curves (where the 95\% curve follows the Master Curve). This, combined with other results below, shows a general trend where probability bounds can widen more than expected with higher temperatures. That the results widen at higher temperatures is likely to be an effect of plasticity which could manifest in both the accuracy of the finite element analyses and of the assumptions in the local approach model.

This can be seen more clearly in the results of the irradiated low constraint base material, in Fig. 5 below. For this case the $50 \%$ bound is slightly over-predicted compared to the experimental data and the $95 \%$ much greater than expected. The results do, however, fit the experimental data and show a different shape to the transition curve under low constraint conditions. This is not necessarily surprising as the low constraint cases will have a larger plastic zone and, as the damage is less focused, will provide a larger range between the probability bounds with applied crack driving force. Likewise, when there are increased levels of widely dispersed plasticity at higher temperatures the likelihood of cleavage reduces so that 
the toughness, and hence the DBT shape, will increase at a different rate to high constraint conditions. As such the results show promise, given the range of assumptions here. It is also likely that the assumption to the use of $2 \mathrm{D}$ finite element geometry will have a greater impact for these low constraint conditions.

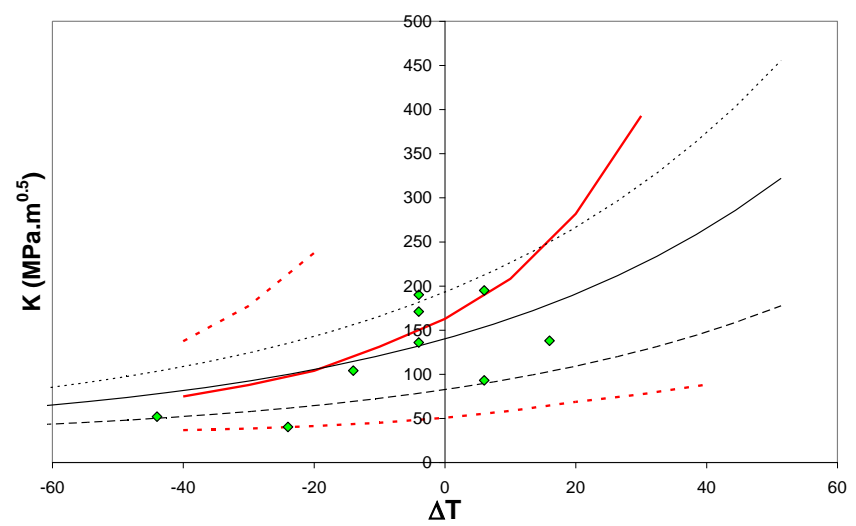

FIGURE 5 - TEMPERATURE DEPENDENCE PREDICTED BY PROPOSED MODEL FOR THE IRRADIATED LOW CONSTRAINT BASE MATERIAL COMPARED TO EXPERIMENTAL DATA AND THE MASTER CURVE.
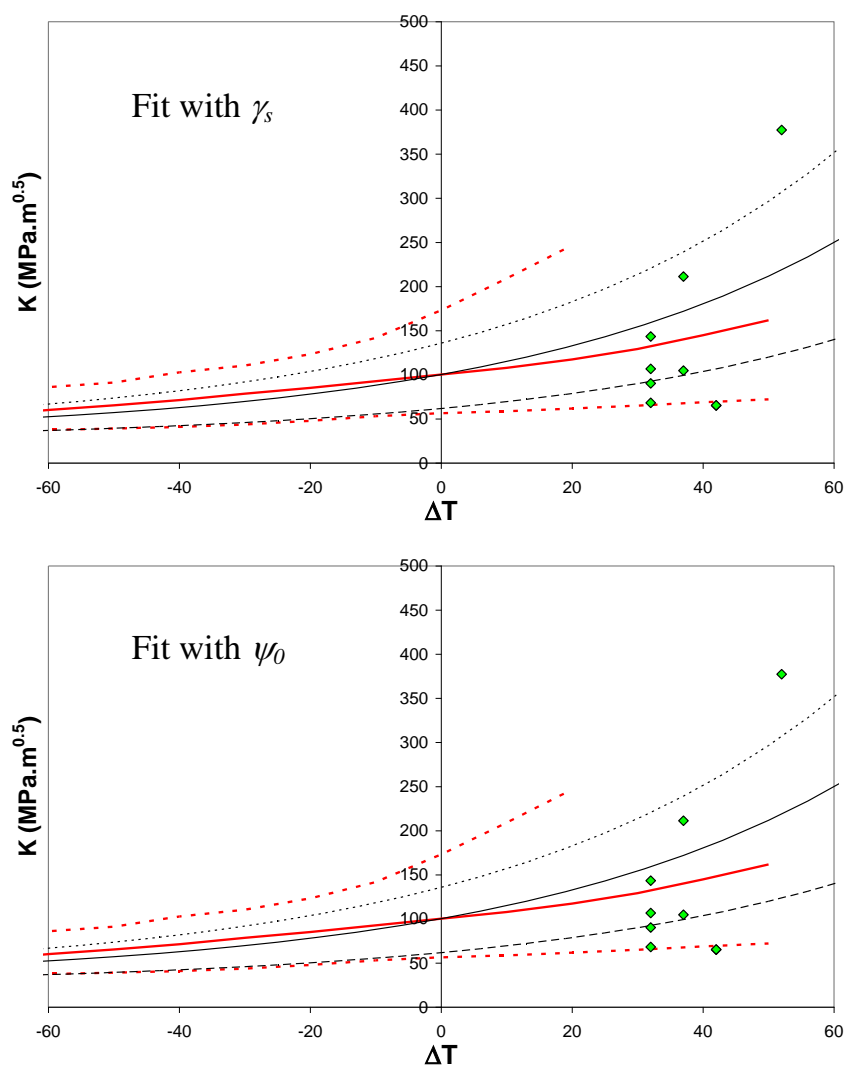

FIGURE 6 - TEMPERATURE DEPENDENCE PREDICTED BY PROPOSED MODEL FOR THE UNIRRADIATED HIGH CONSTRAINT BASE MATERIAL COMPARED TO EXPERIMENTAL DATA AND THE MASTER CURVE.
The same results for the weld material can be seen in Fig. 6 to Fig. 8 below for the unirradiated high constraint case, the irradiated high constraint case and the irradiated low constraint case, respectively. In Fig. 6 the top result is when calibrated by changing $\gamma_{s}$ and fixing $\psi_{0}$, and the bottom figure when changing $\psi_{0}$ and fixing $\gamma_{s}$. However, as the results were seen to be essentially the same for both calibration routes in all cases only one result is show in Fig. 7 and Fig. 8.

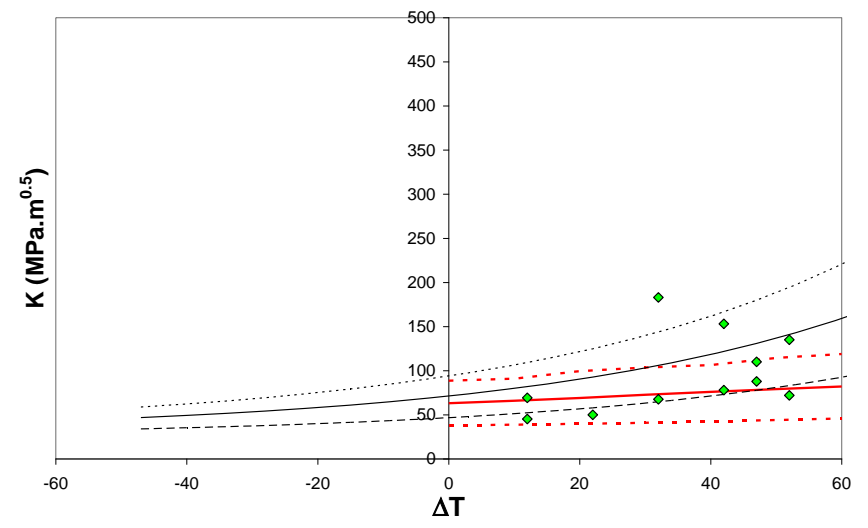

FIGURE 7 - TEMPERATURE DEPENDENCE PREDICTED BY PROPOSED MODEL FOR THE IRRADIATED HIGH CONSTRAINT BASE MATERIAL COMPARED TO EXPERIMENTAL DATA AND THE MASTER CURVE.

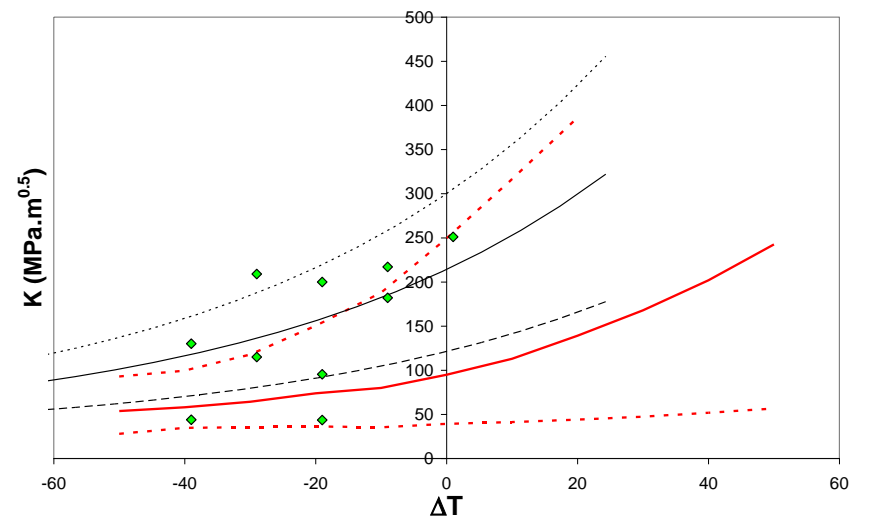

FIGURE 8 - TEMPERATURE DEPENDENCE PREDICTED BY PROPOSED MODEL FOR THE IRRADIATED LOW CONSTRAINT BASE MATERIAL COMPARED TO EXPERIMENTAL DATA AND THE MASTER CURVE.

As for the base material the model presented here provides a good fit of the experimental data in the unirradiated high constraint condition for the weld. The effect of adopting the irradiated material properties is seen to provide too large a toughness shift which causes both the high and low constraint irradiated cases to under-predict the toughness. From comparison to the low constraint condition, the shift is estimated as being $+60{ }^{\circ} \mathrm{C}$ too great. This may be a result of 
the very flat tensile curve adopted and the assumptions used in its definition. Nonetheless, that the results would appear to fit if that shift were included provides some confidence in the proposed model.

It is emphasised that the implementation of the proposed local approach required only the changes of the tensile properties with temperature and irradiation when assessing the changes in the cleavage fracture toughness. Otherwise it used two parameters (the position of the Master Curve was experimentally determined) calibrated at a single temperature for the high constraint, unirradiated case. This indicates that the proposed model can be used predictively, as it is here, without foreknowledge of the toughness values and without the need for further calibration. The proposal is therefore different to other models where, for example, in the Beremin model the value of $\sigma_{u}$ is fit with temperature so that the upswing in results can be obtained.

\section{CONCLUSIONS}

The main conclusions from this paper are:

1) Cleavage toughness predictions in the transition regime made with an improved criterion for carbide rupture that allows micro-structural data, such as initiator particle size distribution, provides a good comparison to both experimental data and Master Curve fits in the unirradiated state for both the base and weld material.

2) On changing the tensile properties in the finite element analyses to consider irradiation the spread of experimental results is well predicted for the base material. This was achieved when only calibrating the approach at one temperature in the unirradiated state.

3) On assessing the effect of irradiation on the weld material the shift in toughness was over-predicted. This is likely to be a result of the assumptions used in defining the material properties which may be more influential given the welds flatter stress-strain curve.

4) When considering the effect of low constraint conditions against high constraint conditions the results show that the low constraint cases exhibit a more rapid DBT transition whilst still following the experimental data.

5) The local approach model presented only requires calibration at a single temperature for it to be used predictively over a range of temperatures, as well as a range of different irradiation and constraint conditions.

\section{ACKNOWLEDGEMENTS}

The support from BNFL to Jivkov is gratefully acknowledged. James would also like to acknowledge assistance from colleagues at AMEC in the FEA and to PERFORM60.

\section{REFERENCES}

[1] A. Pineau, Development of the local approach to fracture over the past 25 years: theory and applications. Int J Fract
138 (2006) 139-166.

[2] F.M. Beremin, A local criterion for cleavage fracture of a nuclear pressure vessel steel. Metal Trans 14A (1983) 2277-2287.

[3] F. Mudry, A local approach to cleavage fracture. Nucl Eng Design 105 (1987) 65-76.

[4] F. Minami, A. Bruckner-Foit, D. Munz, B. Trolldenier, Estimation procedure for the Weibuli parameters used in the local approach. Int J Fract 54 (1992) 197-210.

[5] C. Ruggieri, R.H. Dodds Jr., A transferability model for brittle fracture including constraint and ductile tearing effects: a probabilistic approach. Int J Fract 79 (1996) 309340.

[6] X. Gao, R.H. Dodds Jr., Constraint effects on the ductile-tobrittle transition temperature of ferritic steels: a Weibull stress model. Int J Fract 102 (2000) 43-69.

[7] J.P. Petti, R.H. Dodds Jr., Calibration of the Weibull stress scale parameter, $\sigma_{\mathrm{u}}$, using the Master Curve. Eng Fract Mech 72 (2005) 91-120.

[8] B. Wasiluk, J.P. Petti, R.H. Dodds Jr., Temperature dependence of Weibull stress parameters: Studies using the Euro-material. Eng Fract Mech 73 (2006) 1046-1069.

[9] P. Akbarzadeh, S. Hadidi-Moud, A.M. Goudarzi, Global equations for Weibull parameters in a ductile-to-brittle transition regime. Nucl Eng Design 239 (2009) 11861192.

[10] Y. Cao, H. Hui, G. Wang, F.-Z. Xuan, Inferring the temperature dependence of Beremin cleavage model parameters from the Master Curve. Nucl Eng Design 241 (2011) 39-45.

[11] G. Xiaosheng, G. Zhang, T.S. Srivatsan, A probabilities model for prediction of cleavage fracture in the ductile-tobrittle transition region and the effect of temperature on model parameters. Mater Sci Eng 415A (2006) 264-272.

[12] M. Kroon, J. Faleskog, H. Oberg, A probabilistic model for cleavage fracture with a length scale - Parameter estimation and predictions of growing crack experiments. Eng Fract Mech 75 (2008) 2398-2417.

[13] S.R. Bordet, A.D. Karstensen, D.M. Knowles, C.S. Wiesner, A new statistical local criterion for cleavage fracture of steel. Part I: model presentation. Eng Fract Mech 72 (2005) 435-452.

[14] J. Hohe, V. Hardenacke, S. Luckow, D. Siegele, An enhanced probabilistic model for cleavage fracture assessment accounting for local constraint effects. Eng Fract Mech 77 (2010) 3573-3591.

[15] A.P. Jivkov, D.P.G. Lidbury, P. James, Assessment of local approach methods for predicting end-of-life toughness of RPV steels. In Proc PVP2011 (2011) paper 57546, Baltimore, USA.

[16] J. Hohe, V. Friedmann, J. Wenck, D. Siegele, Assessment of the role of micro defect nucleation in probabilistic modeling of cleavage fracture, Eng Fract Mech 75 (2008) 3306-3327.

[17] K. Wallin, T. Saario, K. Torronen, Statistical model for 
carbide induced brittle fracture in steel. Metals Sci 18 (1984) 13-16.

[18] L.C.A. Folch, F.M. Burdekin, Application of coupled brittle-ductile model to study correlation between Charpy energy and fracture toughness values. Eng Fract Mech 63 (1999) 57-80.

[19] G. Bernauer, W. Brocks, W. Schmitt, Modifications of the Beremin model for cleavage fracture in the transition region of a ferritic steel. Eng Fract Mech 64 (1999) 305325.

[20] A. Pineau, Modeling ductile to brittle fracture transition in steels - micromechanical and physical challenges. Int $\mathbf{J}$ Fract 150 (2008) 129-156.

[21] PERFORM60 online data-base (restricted access), https://perfect.hzdr.de/perform_database.html

[22] ASTM E 1921-05, Standard Test Method for Determination of Reference Temperature T0 for Ferritic Steels in the Transition Range. ASTM, 2005.

[23] S.R. Ortner, J. Duff, Characterisation of Euro 'A' reference steel for application of $\mathrm{EOH}$ model of brittle fracture. Nexia Solutions, Technical Report, 2005.

[24] S.R. Ortner, Fractography of P141 weld metal. National Nuclear Laboratory, Technical Report SPR04782/06/10 $/ 12,2012$.

[25] N. Chapeau, Characterisation of second phase particles in a ferritic steel: Size distribution and density. The University of Manchester (Internship), Technical Report, 2011.

[26] ABAQUS 6.11, DS Simulia Coorp, 2011.

[27] A. Hung, I. Yarovsky, J. Muscat, S. Russo, I. Snook, R.O. Watts, First-principles study of metallic iron interfaces, Surface Science 501: 261-269, 2002.

[28] A.P. Jivkov and P.M. James, Cleavage modeling with experimental particle size distribution and novel particle failure criterion, 13th Int. Conf. on Fracture, 2013. 